\title{
Robust Monotonically Convergent Iterative Learning Control for Discrete-Time Systems via Generalized KYP Lemma
}

\author{
Jian Ding and Huizhong Yang \\ Key Laboratory of Advanced Process Control for Light Industry (Ministry of Education), Jiangnan University, Wuxi 214122, China \\ Correspondence should be addressed to Jian Ding; b111905007@vip.jiangnan.edu.cn
}

Received 24 January 2014; Accepted 12 May 2014; Published 29 May 2014

Academic Editor: Chengjian Zhang

Copyright ( 2014 J. Ding and H. Yang. This is an open access article distributed under the Creative Commons Attribution License, which permits unrestricted use, distribution, and reproduction in any medium, provided the original work is properly cited.

\begin{abstract}
This paper addresses the problem of P-type iterative learning control for a class of multiple-input multiple-output linear discretetime systems, whose aim is to develop robust monotonically convergent control law design over a finite frequency range. It is shown that the $2 \mathrm{D}$ iterative learning control processes can be taken as $1 \mathrm{D}$ state space model regardless of relative degree. With the generalized Kalman-Yakubovich-Popov lemma applied, it is feasible to describe the monotonically convergent conditions with the help of linear matrix inequality technique and to develop formulas for the control gain matrices design. An extension to robust control law design against systems with structured and polytopic-type uncertainties is also considered. Two numerical examples are provided to validate the feasibility and effectiveness of the proposed method.
\end{abstract}

\section{Introduction}

The well-known iterative learning control (ILC) algorithm can effectively improve the transient responses and tracking performance for systems that execute the same task over a finite duration repetitively, the key idea of which is to iteratively reduce the tracking error by refining the control input signal based on the information from previous trials [1]. As demonstrated in survey papers [2-6], ILC has attracted considerable research attention in many areas during the past few decades. Extensive applications of ILC have been used for many practical problems coming from, for example, batch processes [7-9], point-to-point control [10, 11], and positioning control [12-15].

In fact, among all types of ILC research issues, both theoretical and practical, robustness and monotonic convergence have been studied as two major topics. Many uncertain factors such as model uncertainties, variable initial conditions, stochastic noises, and packet dropout need to be taken into consideration with regard to robust ILC design. For example, a kind of so-called adaptive ILC has been developed for local Lipschitz continuous (LLC) uncertain nonlinear systems with unknown parameters, and composite energy function (CEF) is usually constructed to facilitate the convergence analysis [2]. Considering the inherent twodimensional (2 D) structure of every ILC process, 2 D system theory has been developed to design ILC based on linear repetitive processes [16-18], Roesser model $[19,20]$, and Fornasini-Marchsini model [21, 22]. Moreover, the robust ILC has been particularly extended to networked control system $[23,24]$ and switched systems $[25,26]$.

To achieve good learning transients, the monotonic convergence is particularly important in ILC design problems. For example, first-order and second-order P-type ILC schemes are used for continuous linear time-invariant (LTI) systems, where the monotonic convergence of tracking error is guaranteed in the sense of Lebesgue-p norm [27]. It is also noticed that the so-called super-vector formulation for discrete-time ILC has been prevalent for monotonic convergence analysis under different appropriate norm topology. In [28], the monotonic convergence analysis for interval ILC systems is presented for discrete-time systems. A gradientbased optimal ILC scheme is proposed for ensuring robust monotonic convergence [29]. A new semisliding window ILC algorithm is developed for discrete-time LTI systems [30]. Recently, by integrating the technique of linear matrix inequality (LMI), the well-established $H_{\infty}$ norm has been used for deriving monotonical convergence conditions that 
can be described as LMIs and formulas for the control law design, and the tracking error can be ensured to converge monotonically in the sense of $\mathscr{L}_{2}$ norm [31-34].

However, the aforementioned monotonically convergent ILC works treat control law design over the complete frequency range which is not practical in many cases. In particular, the reference signal and design specification are often given for a certain frequency range of relevance. This viewpoint motivates the present study. In this paper, an integrated ILC framework is developed for multipleinput multiple-output (MIMO), LTI discrete systems with a relative degree, and the frequency design can be specified over a finite range. This benefits from the well-established generalized Kalman-Yakubovich-Popov (KYP) lemma that can tie together a frequency domain inequality (FDI) over finite frequency range and an LMI. It is shown that monotonic convergence conditions can be described in terms of LMIs, as well as formulas obtained for the control law design. Furthermore, this approach is extended to handling the robust issues for the systems with norm-bounded and polytopictype uncertainties.

Briefly, the paper is organized as follows. Section 2 introduces several useful LMIs. The problem formulation is supplied in Section 3. The convergence performance of the proposed scheme is analyzed in Section 4. Section 5 provides two illustrative examples. Finally, some concluding remarks are given in Section 6.

Throughout this paper, the following notations are employed. $Z^{+}$denotes the set of nonnegative integers. For a matrix $X$, its transpose, complex conjugate transpose, and orthogonal complement are denoted by $X^{T}, X^{*}$, and $X^{\perp}$, respectively. $I$ and 0 are the identity matrix and the zero matrix with appropriate dimensions, respectively. $X>0$ and $X<0$ denote positive definiteness and negative definiteness, respectively. The symbol $(\star)$ represents the transposed elements in a symmetric matrix and $\rho(\cdot)$ denotes the spectral radius of its matrix argument. For matrices $X$ and $Y, X \otimes Y$ denotes the Kronecker product. $q$ is a forward shift operator along the discrete-time axis; that is, $q x(k)=x(k+1)$.

\section{Preliminary Knowledge}

Before presenting the main results, the following well-known results are briefly introduced in this section.

Lemma 1 (Schur complement, [35]). Given a symmetric matrix $S=\left[\begin{array}{ll}S_{11} & S_{12} \\ S_{21} & S_{22}\end{array}\right], S_{11}$ and $S_{22}$ are square, and then the following inequalities are equivalent:

(1) $S<0$

(2) $S_{11}<0$ and $S_{22}-S_{12}^{T} S_{11}^{-1} S_{12}<0$

(3) $S_{22}<0$ and $S_{11}-S_{12} S_{22}^{-1} S_{12}^{T}<0$.

Lemma 2 (see [36]). Assume $X, Y$, and $Z=Z^{T}$ are real matrices with appropriated dimensions. Then for any matrix $\Sigma$ satisfying $\Sigma^{T} \Sigma \leq I$, the following inequality:

$$
Z+X \Sigma Y+Y^{T} \Sigma^{T} X^{T}<0
$$

TABLE 1

\begin{tabular}{cccc}
\hline & Low frequency & Middle frequency & High frequency \\
\hline$\Theta$ & $|\theta| \leq \theta_{l}$ & $\theta_{1} \leq \theta \leq \theta_{2}$ & $|\theta| \geq \theta_{h}$ \\
\hline$\Psi$ & {$\left[\begin{array}{cc}0 & 1 \\
1 & -2 \cos \left(\theta_{l}\right)\end{array}\right]$} & {$\left[\begin{array}{cc}0 & e^{j \theta_{c}} \\
e^{-j \theta_{c}} & -2 \cos \left(\theta_{w}\right)\end{array}\right]$} & {$\left[\begin{array}{cc}0 & -1 \\
-1 & 2 \cos \left(\theta_{h}\right)\end{array}\right]$} \\
\hline
\end{tabular}

where $\theta_{c}=\left(\theta_{1}+\theta_{2}\right) / 2, \theta_{w}=\left(\theta_{2}-\theta_{1}\right) / 2$.

holds if and only if there exists a scalar $\varepsilon>0$ such that

$$
Z+\varepsilon X X^{T}+\varepsilon^{-1} Y^{T} Y<0
$$

Lemma 3 (see [37]). Assume $X, Y$, and $Z=Z^{T}$ are real matrices with appropriated dimensions. There exists a matrix $W$ such that the following inequality:

$$
Z+X^{T} W Y+Y^{T} W^{T} X<0
$$

holds if and only if the following two inequalities with respect to $W$ are satisfied:

$$
X^{\perp^{T}} Z X^{\perp}<0, \quad Y^{\perp^{T}} Z Y^{\perp}<0
$$

Lemma 4 (generalized KYP lemma, [38]). For a discrete LTI system with transfer function $G(z)$ and frequency response matrix $G\left(e^{j \theta}\right)=\mathbb{C}\left(e^{j \theta} I-\mathbb{A}\right)^{-1} \mathbb{B}+\mathbb{D}$, the following statements are equivalent:

(1) the frequency domain inequality

$$
\begin{array}{r}
{\left[\begin{array}{c}
\left(e^{j \theta} I-\mathbb{A}\right)^{-1} \mathbb{B} \\
I
\end{array}\right]^{*}\left[\begin{array}{cc}
\mathbb{C} & \mathbb{D} \\
0 & I
\end{array}\right]^{*} \Pi\left[\begin{array}{cc}
\mathbb{C} & \mathbb{D} \\
0 & I
\end{array}\right]\left[\begin{array}{c}
\left(e^{j \theta} I-\mathbb{A}\right)^{-1} \mathbb{B} \\
I
\end{array}\right]} \\
\forall \theta \in \Theta
\end{array}
$$

or

$$
\left[\begin{array}{c}
G\left(e^{j \theta}\right) \\
I
\end{array}\right]^{*} \Pi\left[\begin{array}{c}
G\left(e^{j \theta}\right) \\
I
\end{array}\right]<0 \quad \forall \theta \in \Theta
$$

holds for all $e^{j \theta} \in \Lambda(\Phi, \Psi)$, where $\Pi$ is a given real symmetric matrix and

$$
\begin{aligned}
\Lambda(\Phi, \Psi)= & \left\{e^{j \theta} \in C:\left[\begin{array}{c}
e^{j \theta} \\
1
\end{array}\right]^{*} \Phi\left[\begin{array}{c}
e^{j \theta} \\
1
\end{array}\right]=0,\right. \\
& {\left.\left[\begin{array}{c}
e^{j \theta} \\
1
\end{array}\right]^{*} \Psi\left[\begin{array}{c}
e^{j \theta} \\
1
\end{array}\right] \geq 0, \forall \theta \in \Theta\right\}, }
\end{aligned}
$$

where $\Phi=\left[\begin{array}{cc}-1 & 0 \\ 0 & 1\end{array}\right]$ and $\Theta$ denotes the frequency ranges specified by $\Psi$ as shown in Table 1.

(2) There exists Hermitian matrices $P, Q$ such that $Q>0$ and

$$
\begin{gathered}
{\left[\begin{array}{cc}
\mathbb{A} & \mathbb{B} \\
I & 0
\end{array}\right]^{*}(\Phi \otimes P+\Psi \otimes Q)\left[\begin{array}{cc}
\mathbb{A} & \mathbb{B} \\
I & 0
\end{array}\right]} \\
+\left[\begin{array}{cc}
\mathbb{C} & \mathbb{D} \\
0 & I
\end{array}\right]^{*} \Pi\left[\begin{array}{cc}
\mathbb{C} & \mathbb{D} \\
0 & I
\end{array}\right]<0 .
\end{gathered}
$$




\section{Problem Formulation}

3.1. System Description. Consider the following MIMO discrete-time LTI system over $[0, T]$ :

$$
\begin{gathered}
x_{j}(k+1)=A x_{j}(k)+B u_{j}(k), \\
y_{j}(k)=C x_{j}(k)+D u_{j}(k), \\
x_{j}(0)=x_{0}, \quad k \in \mathcal{N}=\{0,1, \ldots, N\},
\end{gathered}
$$

where $j$ is the iteration number, which denotes the $j$ th repetitive operation of the system. The task interval $[0, T]$ is finite and discretized in a set $\mathcal{N}$ that consists of sampled instances $0,1, \ldots, N . x_{j}(k) \in R^{n}$ is the state vector, $u_{j}(k) \in$ $R^{m}$ is the control input vector, and $y_{j}(k) \in R^{p}$ is the output vector. $A, B, C$, and $D$ are constant matrices of appropriate dimensions. $x_{0}$ is the initial condition for each iteration. The relative degree $r(r \geq 0)$ of system (9) can be defined by [34]

(1) $r=0$ if $D \neq 0$;

(2) $r \geq 1$ if it holds that
(a) $C A^{r-1} B \neq 0$;
(b) $D=0$ and $C A^{i} B=0$ for all $i<r-1$.

Let $y_{d}(k)$ denote the reference vector, and then the tracking error on iteration $j$ is

$$
e_{j}(k)=y_{d}(k)-y_{j}(k) .
$$

The control target is to design appropriate control signal $u_{j}(k)$ and present some LMI conditions such that the system output can converge monotonically to the reference trajectory $y_{d}(k)$ over a finite frequency range when the iteration number $j$ tends to infinity, even if there exist system uncertainties.

In order to complete the above control task, the following assumptions are imposed on system (9).

Assumption 5. The initial resetting condition is satisfied; that is, $e_{j}(0)=0, \forall j \in Z^{+}$. Without loss of generality, it is considered that $x_{j}(0)=0$.

Remark 6. Obviously, the transfer function matrix from $u_{j}(k)$ to $y_{j}(k)$ can be expressed as

$$
y_{j}(k)=G_{p}(q) u_{j}(k)
$$

where

$$
G_{p}(q)=C(q I-A)^{-1} B+D=:\left[\begin{array}{c|c}
A & B \\
\hline C & D
\end{array}\right]
$$

is usually obtained by discretizing the original continuoustime domain model using a sampling mechanism that consists of a sampler with the sampling interval $T_{s}$ and a zeroorder hold.

Remark 7. The relative degree $r$ is exactly the steps of delay in the output $y_{j}(k)$ in order to have the control input $u_{j}(k)$ appearing. Note that the relative degree of one, that is, $D=0$ and $C B \neq 0$, is usually considered in the literature for discretetime ILC [5].
3.2. Design of ILC. In this section, the ILC law is introduced as follows:

$$
u_{j+1}(k)=u_{j}(k)+L(q) e_{j}(k),
$$

where $L(q)$ denotes an $m \times p$ polynomial gain operator to be designed.

Subtract $e_{j}(k)$ from $e_{j+1}(k)$ and then use (11) and (13) to obtain

$$
\begin{aligned}
e_{j+1}(k)-e_{j}(k) & =y_{j}(k)-y_{j+1}(k) \\
& =G_{p}(q)\left[u_{j}(k)-u_{j+1}(k)\right] \\
& =-G_{p}(q) L(q) e_{j}(k)
\end{aligned}
$$

which leads to

$$
e_{j+1}(k)=G_{e}(q) e_{j}(k),
$$

where $G_{e}(q)=I-G_{p}(q) L(q)$.

\section{Convergence Analysis}

4.1. Super-Vector Approach. Using the lifting approach, system (9) and ILC law (13) can be described respectively as

$$
\begin{gathered}
\mathbf{Y}_{j}=\mathbf{G}_{\mathbf{p}} \mathbf{U}_{j}, \\
\mathbf{U}_{j+1}=\mathbf{U}_{j}+\mathbf{L E}_{j},
\end{gathered}
$$

where $\mathbf{U}_{j}, \mathbf{Y}_{j}$, and $\mathbf{Y}_{d}, \mathbf{E}_{j}=\mathbf{Y}_{d}-\mathbf{Y}_{j}$, are the supervectors which are lifted to contain $N$ sampled points, and $\mathbf{G}_{\mathbf{p}}$ and $\mathbf{L}$ are two lower triangular block Toeplitz matrices. The elements of $\mathbf{G}_{\mathbf{p}}$ are the system Markov parameters (or the pulse response coefficients).

Equation (16) gives

$$
\mathbf{E}_{j+1}=\mathbf{G}_{\mathbf{e}} \mathbf{E}_{j},
$$

where $\mathbf{G}_{\mathrm{e}}=I-\mathbf{G}_{\mathbf{p}} \mathbf{L}$. For more details of the developments on (16) and (17), refer to [5].

From (17), the monotonic convergence condition can be simply defined in an appropriate norm topology

$$
\left\|\mathbf{E}_{j+1}\right\| \leq\left\|\mathbf{G}_{\mathbf{e}}\right\|\left\|\mathbf{E}_{j}\right\| .
$$

Remark 8. Clearly, when the state-space model matrices $A, B$, $C$, and $D$ have structured and polytopic-type uncertainties, it is difficult to derive learning gain matrix from condition (18).

4.2. Frequency Domain Approach. The following proposition will be helpful for developing frequency-domain monotonic convergence condition.

Proposition 9 (see [31]). Assume $G_{e}(q)=I-G_{p}(q) L(q)$ is stable and causal, if

$$
\left\|G_{e}\left(e^{j \theta}\right)\right\|_{\infty}<1
$$

and then $\left\|\mathrm{G}_{\mathbf{e}}\right\|_{2}<1$; that is, the monotonic convergence of tracking error $\mathbf{E}_{j}$ can be accomplished in $\mathscr{L}_{2}$ norm. 
With Proposition 9, there exist matrices $\mathbb{A}, \mathbb{B}, \mathbb{C}$, and $\mathbb{D}$ such that $G_{e}(q)$ can be expressed by

$$
\begin{aligned}
G_{e}(q) & =\mathbb{C}(q I-\mathbb{A})^{-1} \mathbb{B}+\mathbb{D} \\
& =:\left[\begin{array}{l|l}
\mathbb{A} & \mathbb{B} \\
\hline \mathbb{C} & \mathbb{D}
\end{array}\right] .
\end{aligned}
$$

It is seen that the condition (19) can be resolved by combining a robust $H_{\infty}$ control theory and the LMI technique. However, condition (19) requires control law design over the entire frequency and is a very strict condition. By utilizing the generalized KYP Lemma, this paper develops monotonically convergent ILC design restricted within a finite frequency range. Accordingly, (19) is replaced by following condition:

$$
\left\|G_{e}\left(e^{j \theta}\right)\right\|_{\infty}<1 \quad \forall \theta \in \Theta
$$

where $\Theta$ denotes a finite frequency range.

Moreover, condition (21) is replaced with

$$
\left\|G_{e}\left(e^{j \theta}\right)\right\|_{\infty}<\gamma \quad \forall \theta \in \Theta, \gamma \in(0,1]
$$

However, in this case, inequality (22) is no longer a standard $H_{\infty}$ problem. To this end, we denote $\gamma=\epsilon_{1} \epsilon_{2}^{-1}$ for scalars $\epsilon_{1}>0$ and $\epsilon_{2}>0$. Then if

$$
\epsilon_{1} \leq \epsilon_{2}
$$

holds, $\gamma \in(0,1]$ follows immediately, and at the same time (22) equivalently becomes

$$
\left\|\bar{G}_{e}\left(e^{j \theta}\right)\right\|_{\infty}<\epsilon_{1} \quad \forall \theta \in \Theta,
$$

where $\bar{G}_{e}\left(e^{j \theta}\right)=\epsilon_{2} G_{e}\left(e^{j \theta}\right)$.

Thus, condition (24) can be viewed as an $H_{\infty}$ problem that is subject to a linear constraint condition (23).

Now with controlled system (15), let us further consider how to solve the condition (24) under the generalized KYP Lemma framework. Consider the frequency response matrix $\bar{G}_{e}\left(e^{j \theta}\right)$ and choose the matrix $\Pi$ of Lemma 4 as

$$
\Pi=\left[\begin{array}{cc}
\epsilon_{1}^{-1} I & 0 \\
0 & -\epsilon_{1} I
\end{array}\right] .
$$

We can get

$$
\bar{G}_{e}\left(e^{j \theta}\right)^{*} \bar{G}_{e}\left(e^{j \theta}\right)<\epsilon_{1}^{2} I \quad \forall \theta \in \Theta .
$$

Obviously, inequality (26) is equivalent to condition (24).

4.2.1. Zero Relative Degree $(r=0)$. Consider first that system (9) has a zero relative degree, resulting in

$$
D \neq 0 \text {. }
$$

Accordingly, the ILC law (13) is applied with the following gain operator:

$$
L(q) \equiv L,
$$

where $L$ is an $m \times p$ matrix to be determined.

Moreover, it is easy to see that $G_{e}(q)$ and $\bar{G}_{e}(q)$ satisfy $(20)$ because it can be modeled by

$$
G_{e}(q)=\left[\begin{array}{c|c}
A & -B L \\
\hline C & I-D L
\end{array}\right], \quad \bar{G}_{e}(q)=\left[\begin{array}{c|c}
A & -\epsilon_{2} B L \\
\hline C & \epsilon_{2}(I-D L)
\end{array}\right] .
$$

Now with Lemma 4 and (29), the following theorem can be presented.

Theorem 10. Consider the ILC system (9) and (13) satisfying $r=0$ and Assumption 5, and the gain operator matrix $L(q)$ is defined by (28). Then, $\left\|\mathbf{E}_{j}\right\|_{2}$ converges monotonically to zero over the low frequency range $|\theta| \leq \theta_{l}$ when $j \rightarrow \infty$, if there exist scalars $\epsilon_{1}>0, \epsilon_{2}>0$ and matrices $\widehat{P}>0, \widehat{Q}>0, R, X$ satisfying (23) and the following LMI:

$$
\left[\begin{array}{cccc}
-\widehat{P} & \star & \star & \star \\
\widehat{Q}-R & \widehat{P}-2 \cos \left(\theta_{l}\right) \widehat{Q}+R^{T} A^{T}+A R & \star & \star \\
0 & X^{T} B^{T} & -\epsilon_{1} I & \star \\
0 & C R & \epsilon_{2} I+D X & -\epsilon_{1} I
\end{array}\right] .
$$

If the LMIs of (23) and (30) are feasible, then the gain matrix $L$ is given by

$$
L=-\epsilon_{2}^{-1} X \text {. }
$$

Proof. Applying Lemma 4 gives that condition (26) holds if there exist symmetric matrices $P$ and $Q$ such that $Q>0$ and

$$
\begin{gathered}
{\left[\begin{array}{cc}
\mathbb{A} & \mathbb{B} \\
I & 0
\end{array}\right]^{T}(\Phi \otimes P+\Psi \otimes Q)\left[\begin{array}{cc}
\mathbb{A} & \mathbb{B} \\
I & 0
\end{array}\right]} \\
+\left[\begin{array}{cc}
\mathbb{C} & \mathbb{D} \\
0 & I
\end{array}\right]^{T} \Pi\left[\begin{array}{cc}
\mathbb{C} & \mathbb{D} \\
0 & I
\end{array}\right]<0,
\end{gathered}
$$

where $\mathbb{A}=A, \mathbb{B}=-\epsilon_{2} B L, \mathbb{C}=C, \mathbb{D}=\epsilon_{2}(I-D L)$, $\Pi=\left[\begin{array}{cc}\epsilon_{1}^{-1} I & 0 \\ 0 & -\epsilon_{1} I\end{array}\right]$, and $\Psi$ is the only matrix whose block entries depend on the chosen frequency range. Without loss of generality, the low frequency range is considered; that is, $|\theta| \leq \theta_{l}$, which gives that

$$
\Phi \otimes P+\Psi \otimes Q=\left[\begin{array}{cc}
-P & Q \\
Q & P-2 \cos \left(\theta_{l}\right) Q
\end{array}\right] .
$$

Then, (32) becomes

$$
\begin{gathered}
{\left[\begin{array}{cc}
-\mathbb{A}^{T} P \mathbb{A}+Q \mathbb{A}+\mathbb{A}^{T} Q+P-2 \cos \left(\theta_{l}\right) Q & -\mathbb{A}^{T} P \mathbb{B}+Q \mathbb{B} \\
-\mathbb{B}^{T} P \mathbb{A}+\mathbb{B}^{T} Q & -\mathbb{B}^{T} P \mathbb{B}
\end{array}\right]} \\
+\left[\begin{array}{cc}
\epsilon_{1}^{-1} \mathbb{C}^{T} \mathbb{C} & \epsilon_{1}^{-1} \mathbb{C}^{T} \mathbb{D} \\
\epsilon_{1}^{-1} \mathbb{D}^{T} \mathbb{C} & \epsilon_{1}^{-1} \mathbb{D}^{T} \mathbb{D}-\epsilon_{1} I
\end{array}\right]<0 .
\end{gathered}
$$

The condition of (34) cannot, however, be directly applied to control law design since it involves product terms $\mathbb{A}^{T} P \mathbb{B}$ and $Q \mathbb{B}$. 
To separate the matrices $P$ and $Q$ from the process model matrices, rewrite (34) as

$$
\begin{aligned}
& {\left[\begin{array}{lll}
A^{T} & I & 0 \\
\mathbb{B}^{T} & 0 & I
\end{array}\right]\left[\begin{array}{ccc}
-P & Q & 0 \\
Q & P-2 \cos \left(\theta_{l}\right) Q+\epsilon_{1}^{-1} \mathbb{C}^{T} \mathbb{C} & \epsilon_{1}^{-1} \mathbb{C}^{T} \mathbb{D} \\
0 & \epsilon_{1}^{-1} \mathbb{D}^{T} \mathbb{C} & \epsilon_{1}^{-1} \mathbb{D}^{T} \mathbb{D}-\epsilon_{1} I
\end{array}\right]} \\
& \times\left[\begin{array}{cc}
\mathbb{A} & \mathbb{B} \\
I & 0 \\
0 & I
\end{array}\right]<0 .
\end{aligned}
$$

To apply the result of Lemma 3, we can set $Z=$

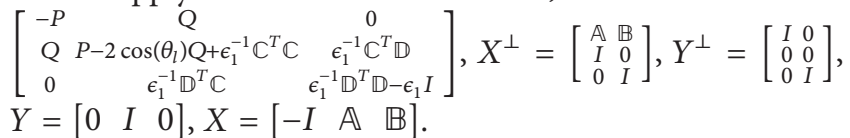

$$
\left[\begin{array}{ccc}
-P & Q-W & 0 \\
Q-W^{T} & P-2 \cos \left(\theta_{l}\right) Q+\epsilon_{1}^{-1} C^{T} C+\mathbb{A}^{T} W+W^{T} \mathbb{A} & \epsilon_{1}^{-1} C^{T} \mathbb{D}+W^{T} \mathbb{B} \\
0 & \epsilon_{1}^{-1} \mathbb{D}^{T} \mathbb{C}+\mathbb{B}^{T} W & \epsilon_{1}^{-1} \mathbb{D}^{T} \mathbb{D}-\epsilon_{1} I
\end{array}\right]<0
$$

Applying the Schur's complement to (38) and inserting $\mathbb{A}=A, \mathbb{B}=-\epsilon_{2} B L, \mathbb{C}=C$, and $\mathbb{D}=\epsilon_{2}(I-D L)$ give that

$$
\left[\begin{array}{cccc}
-P & Q-W & 0 & 0 \\
Q-W^{T} & P-2 \cos \left(\theta_{l}\right) Q+A^{T} W+W^{T} A & -W^{T} \epsilon_{2} B L & C^{T} \\
0 & -\epsilon_{2} L^{T} B^{T} W & -\epsilon_{1} I & \epsilon_{2}(I-D L)^{T} \\
0 & C & \epsilon_{2}(I-D L) & -\epsilon_{1} I
\end{array}\right]<0,
$$

pre- and postmultiplying this last inequality by $\operatorname{diag}\left\{R^{T}\right.$, $\left.R^{T}, I, I\right\}$ and $\operatorname{diag}\{R, R, I, I\}$ to obtain

$$
\left[\begin{array}{cccc}
-R^{T} P R & R^{T}(Q-W) R & 0 & 0 \\
R^{T}\left(Q-W^{T}\right) R & R^{T}\left(P-2 \cos \left(\theta_{l}\right) Q+A^{T} W+W^{T} A\right) R & -R^{T} W^{T} \epsilon_{2} B L & R^{T} C^{T} \\
0 & -\epsilon_{2} L^{T} B^{T} R W & -\epsilon_{1} I & \epsilon_{2}(I-D L)^{T} \\
0 & C R & \epsilon_{2}(I-D L) & -\epsilon_{1} I
\end{array}\right]<0 .
$$

Finally, introduce the change of variables:

$$
X=-\epsilon_{2} L, \quad W=R^{-1}, \quad \widehat{P}=R^{T} P R, \quad \widehat{Q}=R^{T} Q R
$$

giving immediately that (40) is equivalent to the LMI of (30) and the proof is complete.

Next it will be shown that Theorem 10 can be further developed to address system (9) with structured uncertainty matrices of the form:

$$
G_{p}(q)=\left[\begin{array}{c|c}
A+\Delta A & B+\Delta B \\
\hline C+\Delta C & D+\Delta D
\end{array}\right]
$$

where $\Delta A, \Delta B, \Delta C$, and $\Delta D$ represent admissible uncertainties which are assumed to satisfy

$$
\left[\begin{array}{cc}
\Delta A & \Delta B \\
\Delta C & \Delta D
\end{array}\right]=\left[\begin{array}{l}
H_{1} \\
H_{2}
\end{array}\right] \Sigma\left[\begin{array}{ll}
F_{1} & F_{2}
\end{array}\right]
$$

where $H_{1}, H_{2}, F_{1}$, and $F_{2}$ are known matrices of appropriate dimensions, and $\Sigma$ is an unknown matrix satisfying

$$
\Sigma^{T} \Sigma \leq I
$$

In this case, the following robust result can be presented.

Corollary 11. Consider the ILC system (9) and (13) satisfying $r=0$ and Assumption 5. Assume that the plant has uncertain 
matrices described by (43) and (44), and the gain operator matrix $L(q)$ is defined by (28). Then, $\left\|\mathbf{E}_{j}\right\|_{2}$ converges monotonically to zero over the low frequency range $\theta \leq\left|\theta_{l}\right|$ when $j \rightarrow \infty$, if there exist scalars $\epsilon_{1}>0, \epsilon_{2}>0, \epsilon_{3}>0$ and matrices $\widehat{P}>0, \widehat{Q}>0$, and $R, X$ satisfying (23) and the following $L M I$ :

$$
\left[\begin{array}{cc}
-\widehat{P} & \star \\
\widehat{Q}-R & \widehat{P}-2 \cos \left(\theta_{l}\right) \widehat{Q}+R^{T} A^{T}+A R \\
0 & X^{T} B^{T} \\
0 & C R \\
0 & \epsilon_{3} H_{1}^{T} \\
0 & F_{1} R
\end{array}\right.
$$

If the LMIs of (23) and (45) are feasible, then the gain matrix $L$ is given by (31).

Proof. With Theorem 10 applied, this proof can be expressed as the requirement that

$$
\Upsilon+\mathscr{H} \sum \mathscr{F}+\mathscr{H}^{T} \sum \mathscr{F}^{T}<0
$$

where

$$
\begin{aligned}
& \Upsilon \\
& =\left[\begin{array}{cccc}
-\widehat{P} & \star & \star & \star \\
\widehat{Q}-R & \widehat{P}-2 \cos \left(\theta_{l}\right) \widehat{Q}+R^{T} A^{T}+A R & \star & \star \\
0 & X^{T} B^{T} & -\epsilon_{1} I & \star \\
0 & C R & \epsilon_{2} I+D X & -\epsilon_{1} I
\end{array}\right], \\
& \mathscr{H}=\left[\begin{array}{c}
0 \\
H_{1} \\
0 \\
H_{2}
\end{array}\right] \\
& \mathscr{F}=\left[\begin{array}{llll}
0 & F_{1} R & F_{2} X & 0
\end{array}\right] \text {. }
\end{aligned}
$$

With Lemma 2 applied, one has that (46) holds for all $\Sigma$ satisfying $\Sigma^{T} \Sigma \leq I$ if and only if there exists a scalar $\epsilon_{3}>0$ such that

$$
\Upsilon+\epsilon_{3} \mathscr{H}^{T} \mathscr{H}^{T}+\epsilon_{3}^{-1} \mathscr{F}^{T} \mathscr{F}<0 .
$$

It is noted that the above inequality can be rewritten to obtain

$$
\Upsilon+\left[\begin{array}{lll}
\epsilon_{3}^{1 / 2} \mathscr{H} & \epsilon_{3}^{-1 / 2} \mathscr{F}^{T}
\end{array}\right]\left[\begin{array}{l}
\epsilon_{3}^{1 / 2} \mathscr{H}^{T} \\
\epsilon_{3}^{-1 / 2} \mathscr{F}
\end{array}\right]<0
$$

$$
\left.\begin{array}{cccc}
\star & \star & \star & \star \\
\star & \star & \star & \star \\
-\epsilon_{1} I & \star & \star & \star \\
\epsilon_{2} I+D X & -\epsilon_{1} I & \star & \star \\
0 & \epsilon_{3} H_{2}^{T} & -\epsilon_{3} I & \star \\
F_{2} X & 0 & 0 & -\epsilon_{3} I
\end{array}\right]<0 .
$$

Using the Schur complement Lemma, one knows that (49) is equivalent to

$$
\left[\begin{array}{cc}
\Upsilon & \star \\
{\left[\begin{array}{c}
\epsilon_{3}^{1 / 2} \mathscr{H}^{T} \\
\epsilon_{3}^{-1 / 2} \mathscr{F}
\end{array}\right]} & -I
\end{array}\right]<0
$$

Now pre- and post-multiplying (50) by $\operatorname{diag}\{I, I, I, I$, $\left.\epsilon_{3}^{1 / 2} I, \epsilon_{3}^{1 / 2} I\right\}$ result in (45). This proof is completed.

Furthermore, it will be demonstrated that Theorem 10 can be extended to address the case, where the matrices of system (9) are known to lie within a convex bounded uncertain domain $\Omega$ :

$$
\begin{gathered}
\Omega=\left\{(A, B, C, D) \mid(A, B, C, D)=\sum_{i=1}^{N} \tau_{i}\left(A_{i}, B_{i}, C_{i}, D_{i}\right) ;\right. \\
\left.\tau_{i} \geq 0, \sum_{i=1}^{N} \tau_{i}=1\right\} .
\end{gathered}
$$

The following result enables robust ILC design when the model matrices of system (9) belong to a polytope-type uncertain domain $\Omega$.

Corollary 12. Consider the ILC system (9) and (13) satisfying $r=0$ and Assumption 5. Assume that the plant matrices have polytope uncertainty described by (51), and the gain operator matrix $L(q)$ is defined by (28). Then, $\left\|\mathbf{E}_{j}\right\|_{2}$ converges monotonically to zero over the low frequency range $\theta \leq\left|\theta_{l}\right|$ when $j \rightarrow \infty$, if there exist scalars $\epsilon_{1}>0, \epsilon_{2}>0$ and matrices $\widehat{P}_{i}>0, \widehat{Q}_{i}>0, R, X$ satisfying (23) and the following LMIs:

$\left[\begin{array}{cc}\star & \star \\ \star & \star \\ -\epsilon_{1} I & \star \\ \epsilon_{2} I+D_{i} X & -\epsilon_{1} I\end{array}\right]<0, \quad i=1,2, \ldots, N$
If the LMIs of (23) and (52) are feasible, then the gain matrix $L$ is given by (31).
Proof. For all systems of the type of (9) falling within $\Omega$, let $\widehat{P}=\sum_{i=1}^{N} \tau_{i} \widehat{P}_{i}, \widehat{Q}=\sum_{i=1}^{N} \tau_{i} \widehat{Q}_{i}$. Then the LMI of (30) can be 
achieved from the set of LMIs in (52). The remaining of this proof is omitted since it can follow the same lines of the proof of Theorem 10.

4.2.2. Higher-Order Relative Degree $(r \geq 1)$. Consider now that system (9) has a higher-order relative degree of $r \geq 1$, leading to systems of the form (9) with matrices satisfying

$$
D=0, \quad C A^{r-1} B \neq 0, \quad C A^{i} B=0 \quad(i=0, \ldots, r-2) .
$$

In order to compensate for the influence of $r$, the ILC law (13) is considered with an anticipatory gain operator of the form:

$$
L(q)=L_{r} q^{r}
$$

where $L_{r}$ is an $m \times p$ matrix.

Moreover, $G_{e}(q)$ and $\bar{G}_{e}(q)$ can still satisfy (20) as shown in the following:

$$
\begin{gathered}
G_{e}(q)=\left[\begin{array}{cc}
A & -A^{r} B L_{r} \\
C & I-C A^{r-1} B L_{r}
\end{array}\right], \\
\bar{G}_{e}(q)=\left[\begin{array}{cc}
A & -\epsilon_{2} A^{r} B L_{r} \\
C & \epsilon_{2}\left(I-C A^{r-1} B L_{r}\right)
\end{array}\right]
\end{gathered}
$$

or

$$
\begin{gathered}
G_{e}(q)=\left[\begin{array}{cc}
A & -B L_{r} \\
C A^{r} & I-C A^{r-1} B L_{r}
\end{array}\right], \\
\bar{G}_{e}(q)=\left[\begin{array}{cc}
A & -\epsilon_{2} B L_{r} \\
C A^{r} & \epsilon_{2}\left(I-C A^{r-1} B L_{r}\right)
\end{array}\right] .
\end{gathered}
$$

Remark 13. Using the fact that $q(q I-A)^{-1}=I+(q I-A)^{-1} A$ or $q(q I-A)^{-1}=I+A(q I-A)^{-1}$ repetitively yields that $q^{r}(q I-A)^{-1}$ can be expressed as

$$
q^{r}(q I-A)^{-1}=\sum_{l=0}^{r-1} q^{r-1-l} A^{l}+(q I-A)^{-1} A^{r}
$$

or

$$
q^{r}(q I-A)^{-1}=\sum_{l=0}^{r-1} q^{r-1-l} A^{l}+A^{r}(q I-A)^{-1} .
$$

Then $G_{e}(q)$ can be derived as

$$
\begin{aligned}
G_{e}(q) & =I-G_{p}(q) L(q) \\
& =I-C q^{r}(q I-A)^{-1} B L_{r} \\
& =I-C \sum_{l=0}^{r-1} q^{r-1-l} A^{l} B L_{r}-C(q I-A)^{-1} A^{r} B L_{r} \\
& =-C(q I-A)^{-1} A^{r} B L_{r}+I-C A^{r-1} B L_{r}
\end{aligned}
$$

or

$$
\begin{aligned}
G_{e}(q) & =I-G_{p}(q) L(q) \\
& =I-C(q I-A)^{-1} q^{r} B L_{r} \\
& =I-C \sum_{l=0}^{r-1} q^{r-1-l} A^{l} B L_{r}-C(q I-A)^{-1} A^{r} B L_{r} \\
& =-C A^{r}(q I-A)^{-1} B L_{r}+I-C A^{r-1} B L_{r} .
\end{aligned}
$$

Theorem 14. Consider the ILC system (9), (13), and (55) satisfying $r \geq 1$ and Assumption 5, and the gain operator matrix $L(q)$ is defined by (54). Then, $\left\|\mathbf{E}_{j}\right\|_{2}$ converges monotonically to zero over the low frequency range $|\theta| \leq \theta_{l}$ when $j \rightarrow \infty$, if there exist scalars $\epsilon_{1}>0, \epsilon_{2}>0$, and matrices $\widehat{P}>0, \widehat{Q}>0$, $R$, and $X_{r}$ satisfying (23) and the following LMI:

$$
\left[\begin{array}{cccc}
-\widehat{P} & \widehat{Q} & \star & \star \\
\widehat{Q}-R & \widehat{P}-2 \cos \left(\theta_{l}\right) \widehat{Q}+R^{T} A^{T}+A R & \star & \star \\
0 & X_{r}^{T} B^{T} A^{r T} & -\epsilon_{1} I & \star \\
0 & C R & \epsilon_{2} I+C A^{r-1} B X_{r} & -\epsilon_{1} I
\end{array}\right]<0
$$

If the LMIs of (23) and (61) are feasible, then the gain matrix $L_{r}$ is given by

$$
L_{r}=-\epsilon_{2}^{-1} X
$$

Proof. This proof is omitted since it follows identical steps to that of Theorem 10 .

Even if a higher-order relative degree exists, LMIs of (23) and (61) can still be obtained to achieve the monotonic convergence. Since LMI (61) contains product of matrices of the plant $G_{p}(q)$, Theorem 14 cannot be extended like that done in Corollaries 11 and 12. However, it is noted that, for the case $r=1$, the results of Theorem 14 are feasible to deal with the norm-bounded and polytopic-type uncertainties.

Next let us consider only the case where the uncertain model is of the form:

$$
G_{p}(q)=\left[\begin{array}{l|l}
A+\Delta A & B \\
\hline C+\Delta C & 0
\end{array}\right]
$$


The matrices $\Delta A$ and $\Delta C$ represent admissible uncertainties that are assumed to satisfy

$$
\left[\begin{array}{l}
\Delta A \\
\Delta C
\end{array}\right]=\left[\begin{array}{l}
H_{1} \\
H_{2}
\end{array}\right] \Sigma F_{1},
$$

where $H_{1}, H_{2}$, and $F_{1}$ are known constant matrices, and $\Sigma$ is an unknown matrix satisfying $\Sigma^{T} \Sigma \leq I$. Now the effects of norm-bounded uncertainty can be addressed via the following result.
Corollary 15. Consider the ILC system (9), (13), and (55) satisfying $r=1$ and Assumption 5. Assume that the plant has uncertain matrices described by (63) and (64), and the gain operator matrix $L(q)$ is defined by (54). Then, $\left\|\mathbf{E}_{j}\right\|_{2}$ converges monotonically to zero over the low frequency range $\theta \leq\left|\theta_{l}\right|$ when $j \rightarrow \infty$, if there exist scalars $\epsilon_{1}>0, \epsilon_{2}>0, \epsilon_{3}>0$ and matrices $\widehat{P}>0, \widehat{Q}>0, R, X_{r}$ satisfying (23) and the following LMI:

$$
\left[\begin{array}{cc}
-\widehat{P} & \star \\
\widehat{Q}-R & \widehat{P}-2 \cos \left(\theta_{l}\right) \widehat{Q}+R^{T} A^{T}+A R \\
0 & X_{r}^{T} B^{T} A^{T} \\
0 & C R \\
0 & \epsilon_{3} H_{1}^{T} \\
0 & F_{1} R
\end{array}\right.
$$

If the LMIs of (23) and (65) are feasible, then the gain matrix $L_{r}$ is given by (62).

Proof. This proof is omitted since it follows in an identical manner to that of Corollary 11.

Furthermore, the matrices $A$ and $C$ are known to lie within an uncertainty polytope $\Omega_{1}$ where

$$
\Omega_{1}=\left\{(A, C) \mid(A, C)=\sum_{i=1}^{N} \tau_{i}\left(A_{i}, C_{i}\right) ; \tau_{i} \geq 0, \sum_{i=1}^{N} \tau_{i}=1\right\} .
$$

$$
\left.\begin{array}{cccc}
\star & \star & \star & \star \\
\star & \star & \star & \star \\
-\epsilon_{1} I & \star & \star & \star \\
\epsilon_{2} I+C B X & -\epsilon_{1} I & \star & \star \\
0 & \epsilon_{3} H_{2}^{T} & -\epsilon_{3} I & \star \\
F_{1} B X_{r} & 0 & 0 & -\epsilon_{3} I
\end{array}\right]<0 .
$$

The following result is able to address the problems of polytope-type uncertainty.

Corollary 16. Consider the ILC system (9), (13), and (55) satisfying $r=1$ and Assumption 5. Assume that the plant matrices have polytope uncertainty described by (66), and the gain operator matrix $L(q)$ is defined by (54). Then, $\left\|\mathbf{E}_{j}\right\|_{2}$ converges monotonically to zero over the low frequency range $\theta \leq\left|\theta_{l}\right|$ when $j \rightarrow \infty$, if there exist scalars $\epsilon_{1}>0, \epsilon_{2}>0$ and matrices $\widehat{P}_{i}>0, \widehat{Q}_{i}>0, R, X$ satisfying (23) and the following LMIs:

$$
\left[\begin{array}{cccc}
-\widehat{P}_{i} & \widehat{Q}_{i} & \star & \star \\
\widehat{Q}_{i}-R & \widehat{P}_{i}-2 \cos \left(\theta_{l}\right) \widehat{Q}_{i}+R^{T} A_{i}^{T}+A_{i} R & \star & \star \\
0 & X_{r}^{T} B^{T} A_{i}^{T} & -\epsilon_{1} I & \star \\
0 & C_{i} R & \epsilon_{2} I+C_{i} B X_{r} & -\epsilon_{1} I
\end{array}\right]<0
$$

If the LMIs of (23) and (67) are feasible, then the gain matrix $L_{r}$ is given by (62).

Proof. This proof is omitted since it follows identical steps to that of Corollary 12.

Remark 17. Considering the dual transfer function $G_{e}(q)$ that is described by (56), the following result can be obtained in an identical manner to that of Theorem 18.
Theorem 18. Consider the ILC system (9), (13), and (56) satisfying $r \geq 1$ and Assumption 5, and the gain operator matrix $L(q)$ is defined by (54). Then, $\left\|\mathbf{E}_{j}\right\|_{2}$ converges monotonically to zero over the low frequency range $|\theta| \leq \theta_{l}$ when $j \rightarrow \infty$, if there exist scalars $\epsilon_{1}>0, \epsilon_{2}>0$, and matrices $\widehat{P}>0, \widehat{Q}>0$, $R$, and $X_{r}$ satisfying (23) and the following LMI:

$$
\left[\begin{array}{cccc}
-\widehat{P} & \star & \star & \star \\
\widehat{Q}-R & \widehat{P}-2 \cos \left(\theta_{l}\right) \widehat{Q}+R^{T} A^{T}+A R & \star & \star \\
0 & X_{r}^{T} B^{T} & -\epsilon_{1} I & \star \\
0 & C A^{r} R & \epsilon_{2} I+C A^{r-1} B X_{r} & -\epsilon_{1} I
\end{array}\right]<0 .
$$




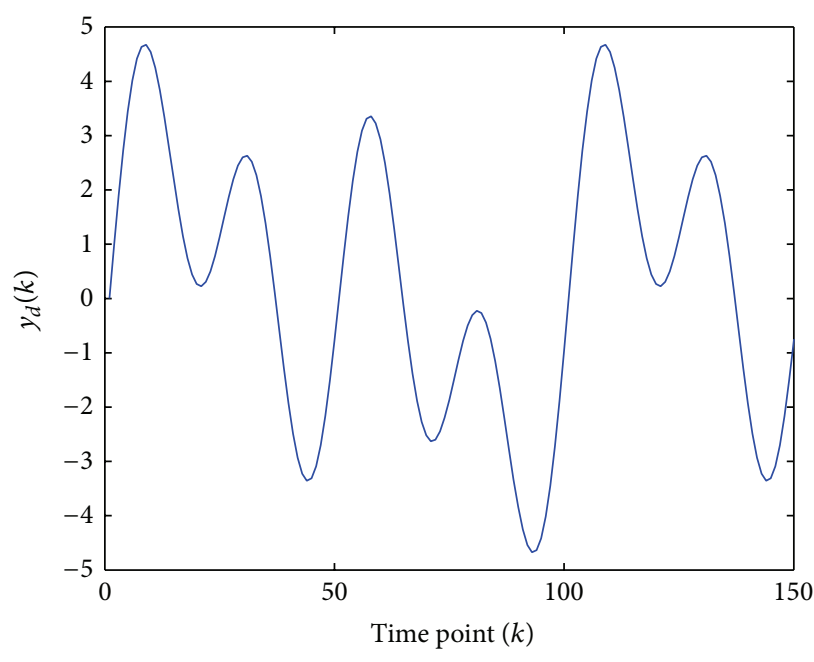

(a)

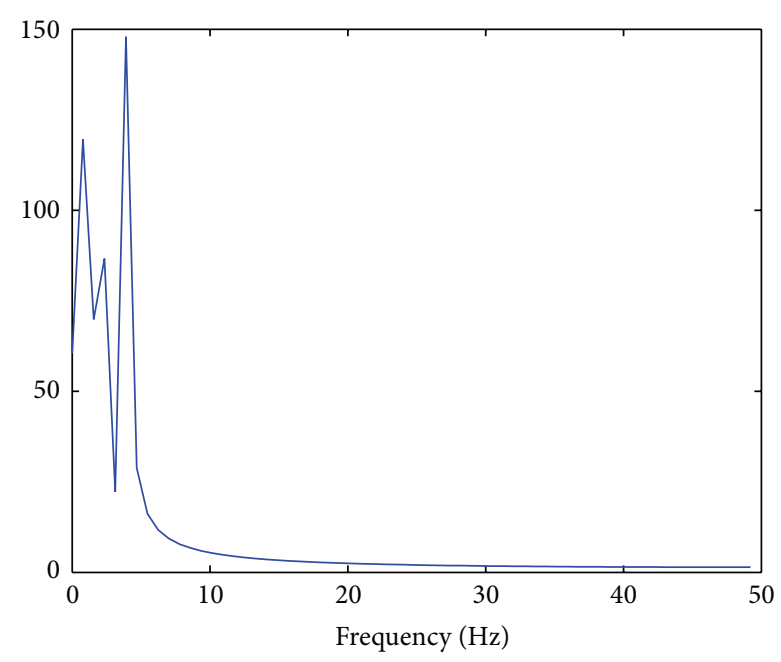

(b)

FIGURE 1: (a) The reference trajectory and (b) the frequency spectrum for the reference trajectory.

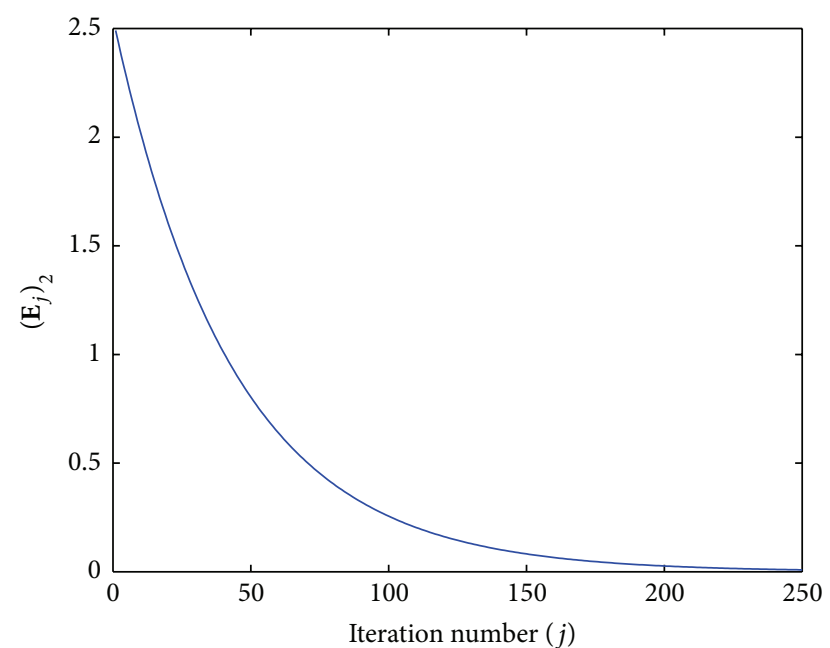

Figure 2: Example 1, $\mathscr{L}_{2}$ norm of tracking error with respect to the iteration number.

If the LMIs of (23) and (68) are feasible, then the gain matrix $L_{r}$ is given by (62).

Based on Theorem 18, the robust results with $r=1$ are omitted since it follows identical steps to those of Corollary 15 and Corollary 16.

\section{Simulation Examples}

Example 1. Consider the following SISO system given by [28]:

$$
\begin{array}{cc}
A=\left[\begin{array}{ccc}
0.72 & 0 & 0 \\
1.0 & -1.04 & -0.81 \\
0 & 0.81 & 0
\end{array}\right], & B=\left[\begin{array}{l}
1 \\
0 \\
0
\end{array}\right], \\
C=\left[\begin{array}{lll}
1 & -0.98 & -1.09
\end{array}\right], \quad D=0 .
\end{array}
$$

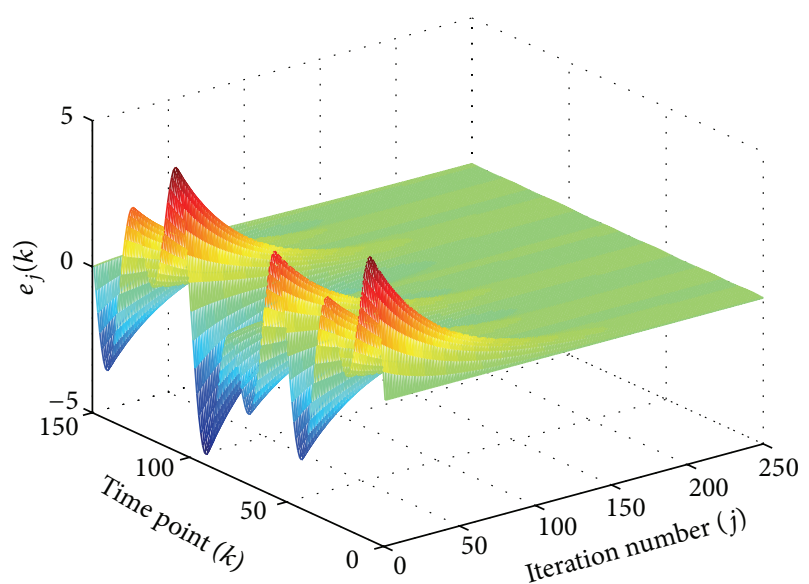

FIgURE 3: Example 1, process of the tracking error along both the iteration axis and the time axis.

Obviously, the considered system has a relative degree of one. Each iteration duration $T$ is $1.5 \mathrm{~s}$ and the sampling frequency is set to $100 \mathrm{~Hz}$. The reference trajectory is shown in Figure 1(a) and associated frequency spectrum in Figure 1(b). Inspecting the amplitudes in the frequency spectrum, it is shown that significant harmonics in the range from 0 to $10 \mathrm{~Hz}$, which can be taken as the low frequency range. And hence $\theta_{l}$ is chosen as $\theta_{l}=0.6284$.

For uncertainties modeled by (64), assume that $H_{1}=$ $\operatorname{diag}\{-0.05,0.1,0.1\}, H_{2}=[-0.12,0.15,0.15], F_{1}=I$, and $\Sigma=\operatorname{diag}\left\{\sigma_{1}, \sigma_{2}, \sigma_{3}\right\}$, where $\sigma_{1}, \sigma_{2}$, and $\sigma_{3}$ vary randomly between -1 and 1 . Then apply Corollary 15 and use the LMI solver "feasp" in the Matlab toolbox to obtain $L=0.0304$.

The simulation results are shown in Figures 2, 3, and 4. From these three figures, it is clearly demonstrated that the tracking error converges monotonically to zero along the iteration axis. 


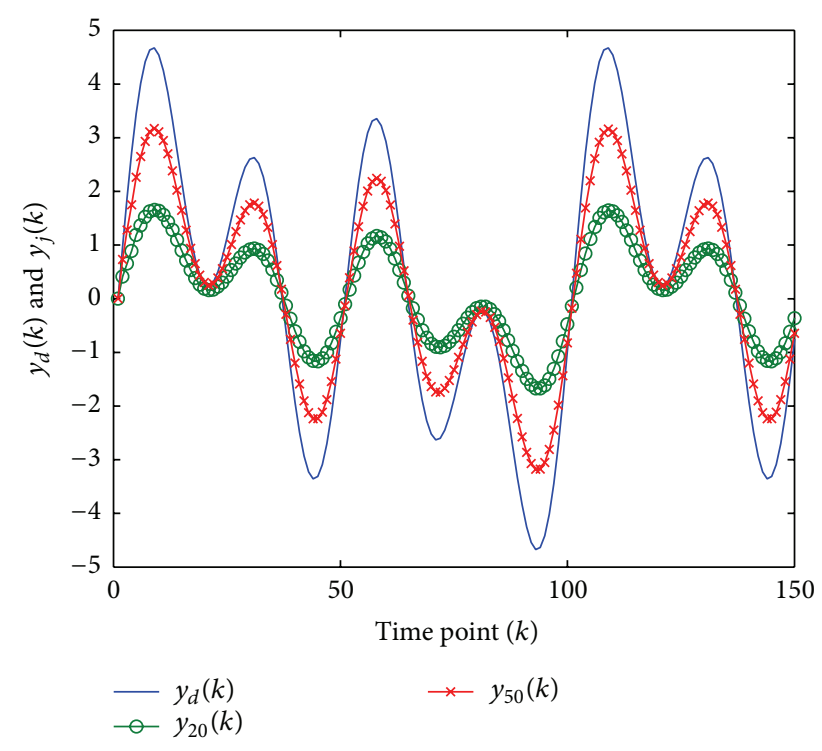

FIgURE 4: Example 1, the reference $y_{d}(k)$ and output $y_{j}(k)$ for $j=20$ and $j=50$.

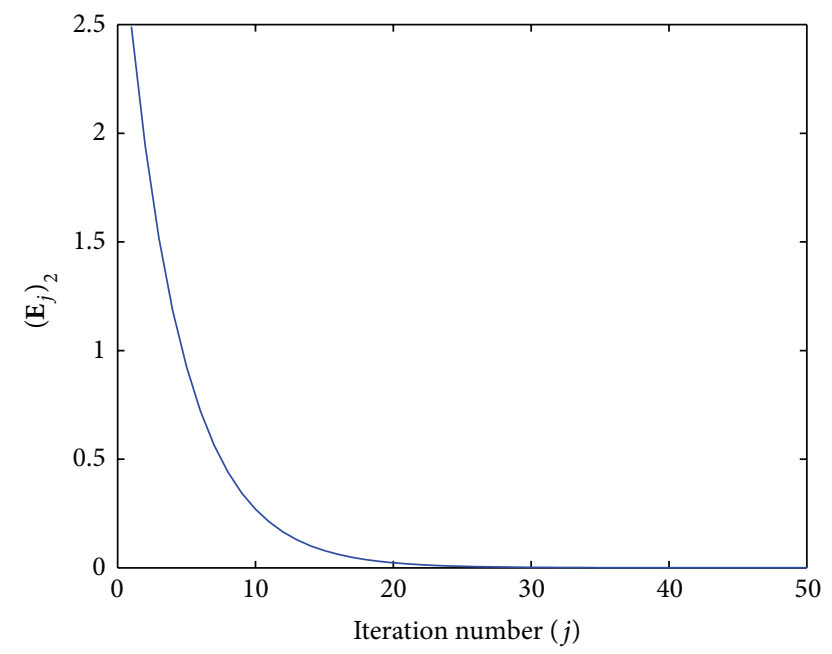

Figure 5: Example 2, $\mathscr{L}_{2}$ norm of tracking error with respect to the iteration number.

Example 2. In this example, the system is considered with matrices given by

$$
\begin{gathered}
A=\left[\begin{array}{ccc}
0.72 & 0 & 0 \\
1.0 & -1.04 & -0.81 \\
0 & 0.81+a & 0
\end{array}\right], \quad B=\left[\begin{array}{l}
1 \\
0 \\
0
\end{array}\right], \\
C=\left[\begin{array}{lll}
1 & -0.98+c-1.09
\end{array}\right], \quad D=0,
\end{gathered}
$$

where $a$ and $c$ are uncertain parameters in the form of $(a, c)=$ $\tau_{1}\left(a_{1}, c_{1}\right)+\tau_{2}\left(a_{2}, c_{2}\right)$. Here, $\left(a_{1}, c_{1}\right)=(-0.4,-0.4),\left(a_{2}, c_{2}\right)=$ $(0.4,0.4)$, and $\tau_{1}+\tau_{2}=1$, and $\tau_{1}$ and $\tau_{2}$ are uncertain variables lying in the interval $[0,1]$.

The simulation condition is performed identically to Example 1. Then apply Corollary 16 to such a system and solve

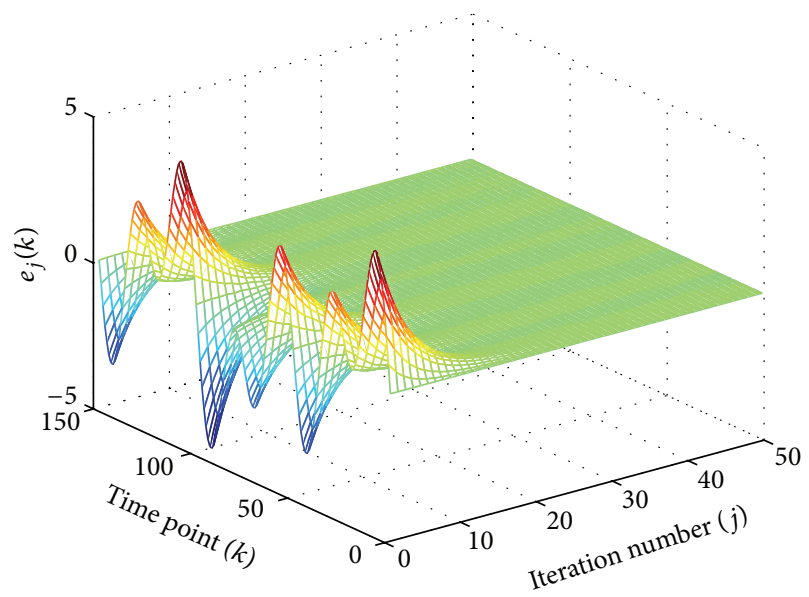

FIGURE 6: Example 2, process of the tracking error along both the iteration axis and the time axis.

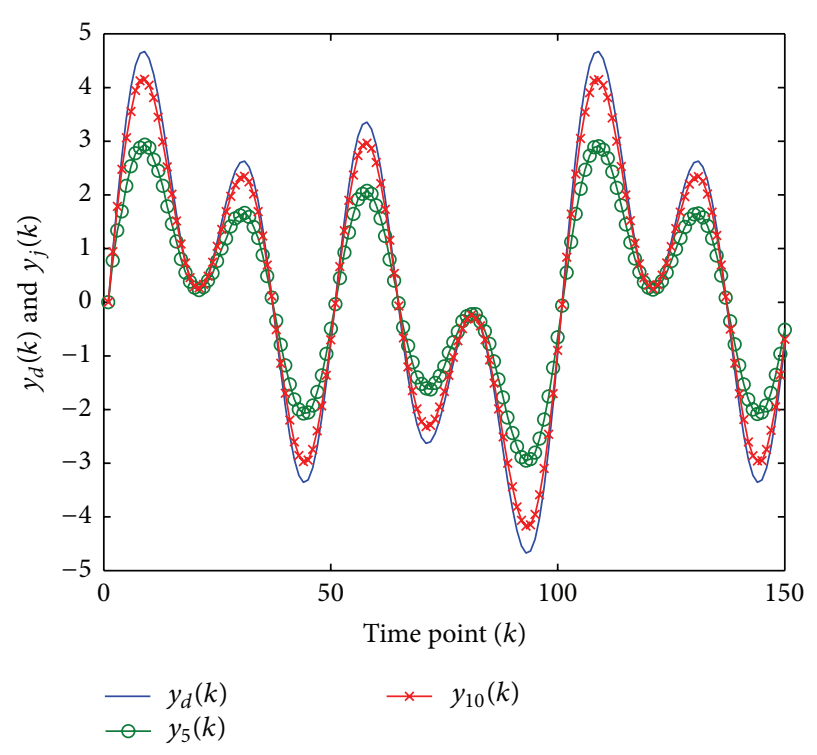

Figure 7: Example 2, the reference $y_{d}(k)$ and output $y_{j}(k)$ for $j=5$ and $j=10$.

LMIs (23) and (67) to obtain $L_{r}=0.3326$. The simulation results are shown in Figures 5, 6, and 7, from which it is seen that the tracking error also decays monotonically to zero along the iteration axis.

\section{Conclusion}

This paper deals with tracking problem of uncertain MIMO discrete-time systems with a relative degree. Based on the idea of generalized Kalman-Yakubovich-Popov lemma, the proposed ILC scheme achieves robust monotonically convergent control law design over a finite frequency range, and sufficient conditions in terms of LMIs have been developed. The effectiveness of the controller design is validated through two numerical examples. 


\section{Conflict of Interests}

The authors declare that there is no conflict of interests regarding the publication of this paper.

\section{Acknowledgment}

This work was supported by the National Natural Science Foundation of China (nos. 61273070 and 61203092).

\section{References}

[1] S. Arimoto, S. Kawamura, and F. Miyazaki, "Bettering operation of robotics by learning," Journal of Robotic Systems, vol. 12, no. 2, pp. 123-140, 1984.

[2] J. X. Xu, "A survey on iterative learning control for nonlinear systems," International Journal of Control, vol. 84, no. 7, pp. 1275-1294, 2011.

[3] H. S. Ahn, Y. Q. Chen, and K. L. Moore, "Iterative learning control: Brief survey and categorization," IEEE Transactions on Systems, Man and Cybernetics C: Applications and Reviews, vol. 37, no. 6, pp. 1099-1121, 2007.

[4] Y. Wang, F. Gao, and F. J. Doyle III, "Survey on iterative learning control, repetitive control, and run-to-run control," Journal of Process Control, vol. 19, no. 10, pp. 1589-1600, 2009.

[5] D. A. Bristow, M. Tharayil, and A. G. Alleyne, "A survey of iterative learning control: A learning-based method for highperformance tracking control," IEEE Control Systems Magazine, vol. 26, no. 3, pp. 96-114, 2006.

[6] X. E. Ruan, K. H. Park, and Z. Z. Bien, "Retrospective review of some iterative learning control techniques with a comment on prospective long-term learning," Control Theory and Applications, vol. 29, no. 8, pp. 966-973, 2012.

[7] J. Li, Y. Tian, and C. Minsen, "An integrated iterative learning control strategy with model identification and dynamic Rparameter for batch processes," Journal of Process Control, vol. 23, no. 9, pp. 1332-1341, 2013.

[8] H. Ahn, K. Lee, M. Kim, and J. Lee, "Control of a reactive batch distillation process using an iterative learning technique," Korean Journal of Chemical Engineering, vol. 31, no. 1, pp. 6-11, 2014.

[9] X. Yu, Z. Xiong, D. Huang, and Y. Jiang, "Model-based iterative learning control for batch processes using generalized hinging hyperplanes," Industrial and Engineering Chemistry Research, vol. 52, no. 4, pp. 1627-1634, 2013.

[10] C. T. Freeman, "Constrained point-to-point iterative learning control with experimental verification," Control Engineering Practice, vol. 20, no. 5, pp. 489-498, 2012.

[11] C. T. Freeman and Y. Tan, "Iterative learning control with mixed constraints for point-to-point tracking," IEEE Transactions on Control Systems Technology, vol. 21, no. 3, pp. 604-616, 2013.

[12] B. E. Helfrich, C. Lee, D. A. Bristow et al., "Combined $H_{\infty}$ feedback control and iterative learning control design with application to nanopositioning systems," IEEE Transactions on Control Systems Technology, vol. 18, no. 2, pp. 336-351, 2010.

[13] G. Parmar, K. Barton, and S. Awtar, "Large dynamic range nanopositioning using iterative learning control," Precision Engineering, 2013.

[14] J. X. Xu, D. Huang, V. Venkataramanan, and T. C. T. Huynh, "Extreme precise motion tracking of piezoelectric positioning stage using sampled-data iterative learning control," IEEE
Transactions on Control Systems Technology, vol. 21, no. 4, pp. 1432-1439, 2013.

[15] D. Huang, J. X. Xu, V. Venkataramanan, and C. T. Huynh, "High-performance tracking of piezoelectric positioning stage using current-cycle iterative learning control with gain scheduling," IEEE Transactions on Industrial Electronics, vol. 61, no. 2, pp. 1085-1098, 2014.

[16] L. Hladowski, K. Galkowski, Z. Cai, E. Rogers, C. T. Freeman, and P. L. Lewin, "Experimentally supported 2D systems based iterative learning control law design for error convergence and performance," Control Engineering Practice, vol. 18, no. 4, pp. 339-348, 2010.

[17] P. Dabkowski, K. Galkowskiy, E. Rogers, Z. Cai, C. T. Freeman, and P. L. Lewin, "Iterative learning control based on relaxed 2-D systems stability criteria," IEEE Transactions on Control Systems Technology, vol. 21, no. 3, pp. 1016-1023, 2013.

[18] W. Paszke, E. Rogers, K. Gałkowski, and Z. Cai, "Robust finite frequency range iterative learning control design and experimental verification," Control Engineering Practice, vol. 21, no. 10, pp. 1310-1320, 2013.

[19] T. Liu and Y. Wang, "A synthetic approach for robust constrained iterative learning control of piecewise affine batch processes," Automatica, vol. 48, no. 11, pp. 2762-2775, 2012.

[20] Y. Wang, Y. Yang, and Z. Zhao, "Robust stability analysis for an enhanced ILC-based PI controller," Journal of Process Control, vol. 23, no. 2, pp. 201-214, 2013.

[21] L. Wang and W. Dong, "Optimal iterative learning fault-tolerant guaranteed cost control for batch processes in the 2D-FM model," Abstract and Applied Analysis, vol. 2012, Article ID 748981, 21 pages, 2012.

[22] L. Wang, X. Chen, and F. Gao, "An LMI method to robust iterative learning fault-tolerant guaranteed cost control for batch processes," Chinese Journal of Chemical Engineering, vol. 21, no. 4, pp. 401-411, 2013.

[23] R. Wang, X. Ma, and X. Bu, "Robust iterative learning control for a class of linear systems with data dropouts," Mathematical Problems in Engineering, vol. 2013, Article ID 747053, 9 pages, 2013.

[24] L. X. Huang and Y. Fang, "Convergence analysis of wireless remote iterative learning control systems with dropout compensation," Mathematical Problems in Engineering, vol. 2013, Article ID 609284, 9 pages, 2013.

[25] X. Bu, F. Yu, Z. Fu, and F. Wang, "Stability analysis of highorder iterative learning control for a class of nonlinear switched systems," Abstract and Applied Analysis, vol. 2013, Article ID 684642, 13 pages, 2013.

[26] X. Bu, Z. Hou, F. Yu, and Z. Fu, "Iterative learning control for a class of non-linear switched systems," IET Control Theory \& Applications, vol. 7, no. 3, pp. 470-481, 2013.

[27] X. Ruan, Z. Z. Bien, and Q. Wang, "Convergence characteristics of proportional-type iterative learning control in the sense of Lebesgue- $p$ norm," IET Control Theory \& Applications, vol. 6, no. 5, pp. 707-714, 2012.

[28] H. S. Ahn, K. L. Moore, and Y. Chen, "Stability analysis of discrete-time iterative learning control systems with interval uncertainty," Automatica, vol. 43, no. 5, pp. 892-902, 2007.

[29] D. H. Owens, J. J. Hatonen, and S. Daley, "Robust monotone gradient-based discrete-time iterative learning control," International Journal of Robust and Nonlinear Control, vol. 19, no. 6, pp. 634-661, 2009. 
[30] A. Madady and H. R. Reza-Alikhani, "A guaranteed monotonically convergent iterative learning control," Asian Journal of Control, vol. 14, no. 5, pp. 1299-1316, 2012.

[31] D. Meng, Y. Jia, J. Du, and F. Yu, "Robust learning controller design for MIMO stochastic discrete-time systems: an $\mathrm{H}_{\infty}{ }^{-}$ based approach," International Journal of Adaptive Control and Signal Processing, vol. 25, no. 7, pp. 653-670, 2011.

[32] D. Meng, Y. Jia, J. Du, and F. Yu, "Monotonically convergent ILC systems designed using bounded real lemma," International Journal of Systems Science: Principles and Applications of Systems and Integration, vol. 43, no. 11, pp. 2062-2071, 2012.

[33] D. Meng, Y. Jia, J. Du, and F. Yu, "Robust design of a class of time-delay iterative learning control systems with initial shifts," IEEE Transactions on Circuits and Systems I: Regular Papers, vol. 56, no. 8, pp. 1744-1757, 2009.

[34] D. Meng, Y. Jia, J. Du, and F. Yu, "Data-driven control for relative degree systems via iterative learning," IEEE Transactions on Neural Networks, vol. 22, no. 12, pp. 2213-2225, 2011.

[35] S. Boyd, L. Ghaoui, E. Feron, and V. Balakrishnan, Linear Matrix Inequalities in System and Control Theory, SIAM, Philadelphia, $\mathrm{Pa}, \mathrm{USA}, 1994$.

[36] L. Xie, "Output feedback $H_{\infty}$ control of systems with parameter uncertainty," International Journal of Control, vol. 63, no. 4, pp. 741-750, 1996.

[37] P. Gahinet and P. Apkarian, "A linear matrix inequality approach to $H_{\infty}$ control," International Journal of Robust and Nonlinear Control, vol. 4, no. 4, pp. 421-448, 1994.

[38] T. Iwasaki and S. Hara, "Generalized KYP lemma: unified frequency domain inequalities with design applications," IEEE Transactions on Automatic Control, vol. 50, no. 1, pp. 41-59, 2005. 


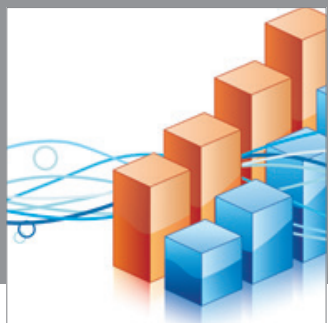

Advances in

Operations Research

mansans

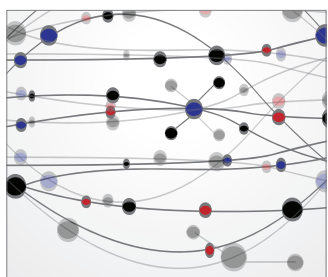

The Scientific World Journal
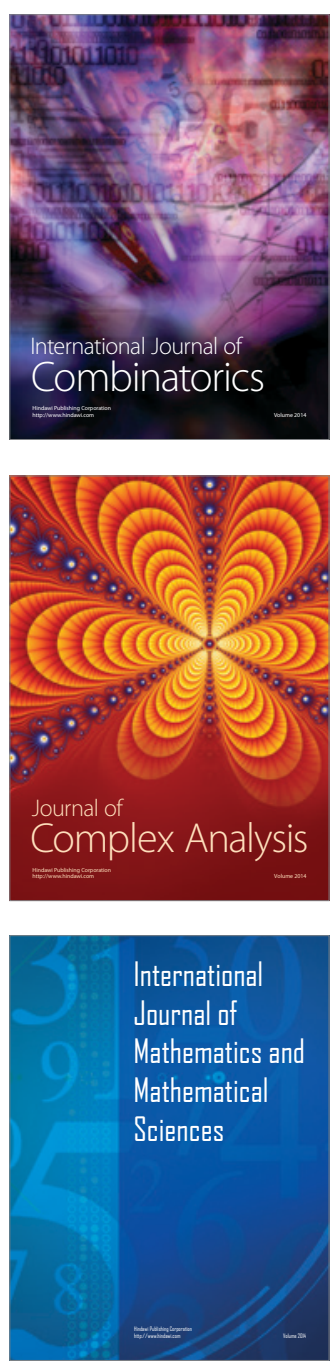
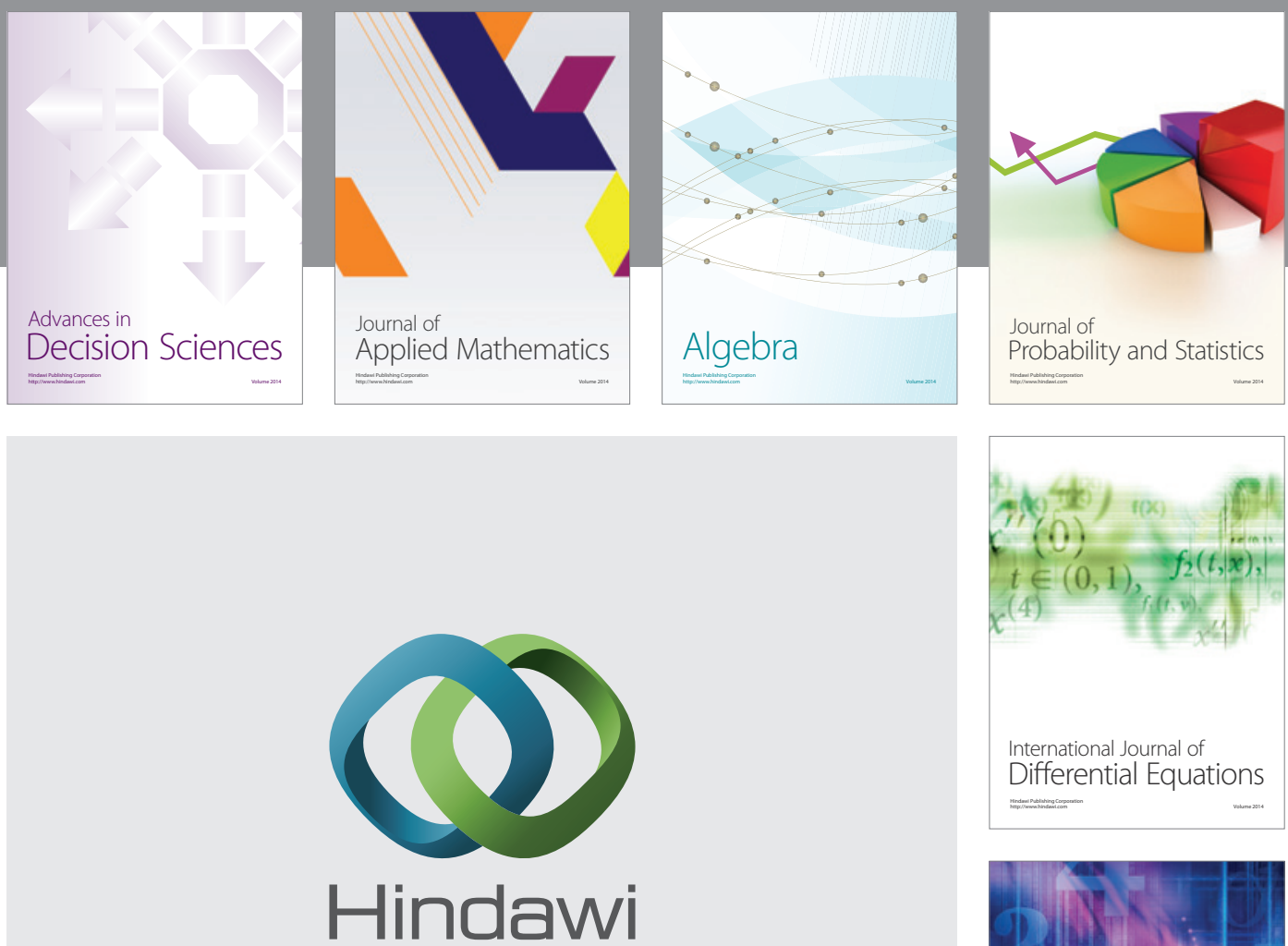

Submit your manuscripts at http://www.hindawi.com
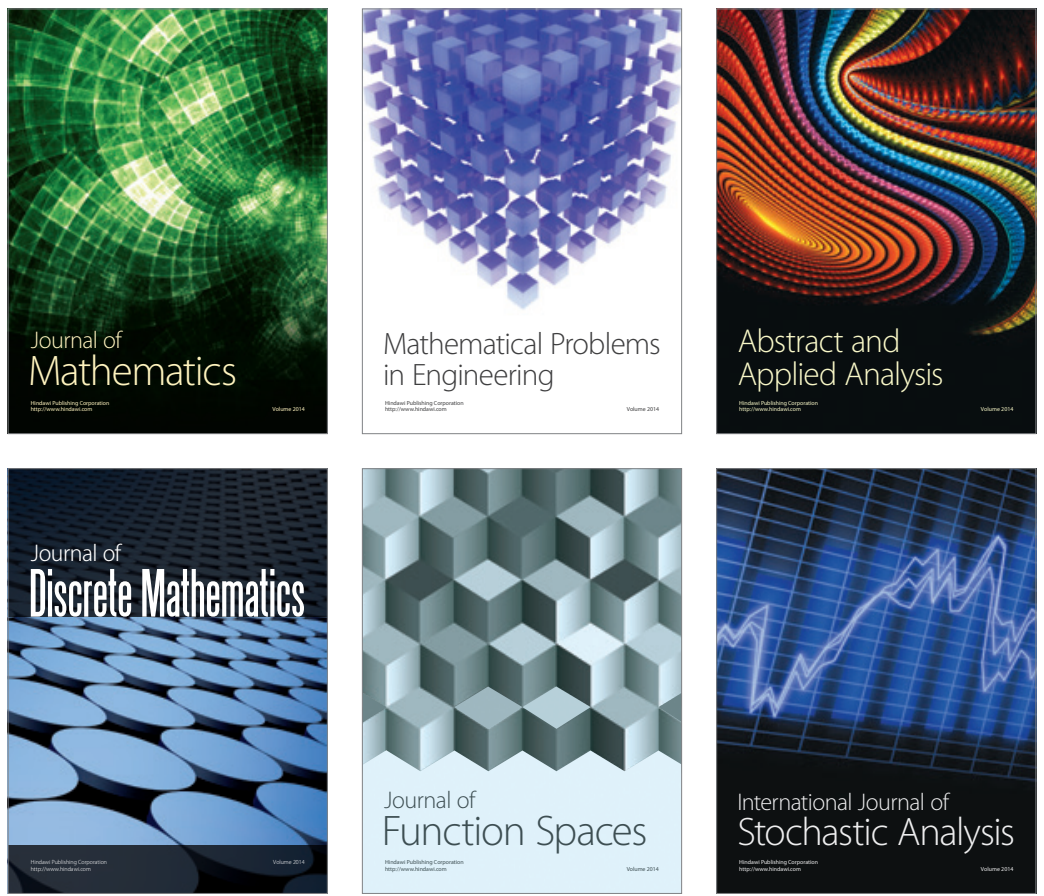

Journal of

Function Spaces

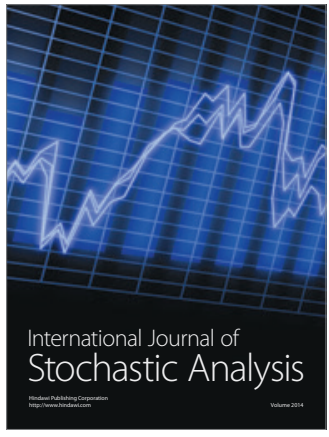

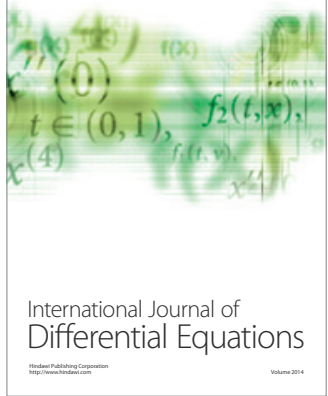
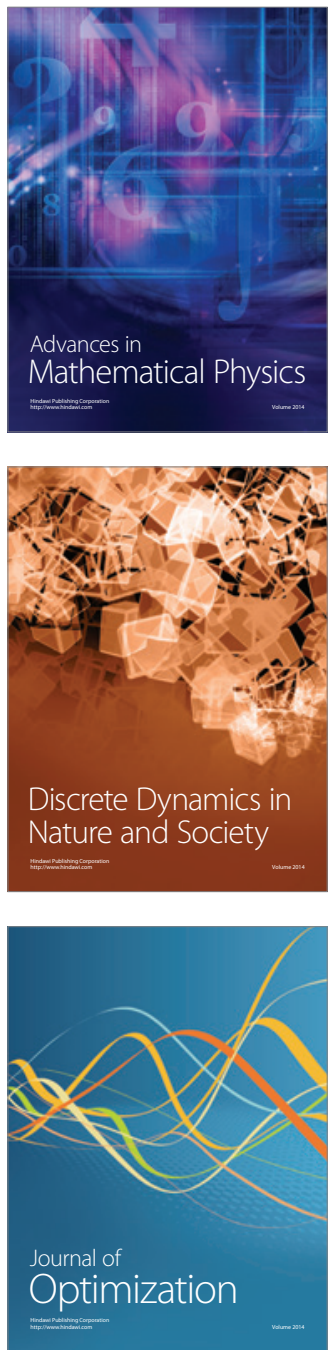